OPEN ACCESS

Edited by:

George Tsiamis,

University of Patras, Greece

Reviewed by:

Carmen Wacher,

National Autonomous University of

Mexico, Mexico

Spyridon Ntougias,

Democritus University of Thrace,

Greece

*Correspondence:

Ming Chen

cm990919@163.com

these authors have contributed equally to this work

Specialty section:

This article was submitted to Systems Microbiology,

a section of the journal

Frontiers in Microbiology

Received: 13 August 2018 Accepted: 14 December 2018 Published: 09 January 2019

Citation:

Li M, Li L, Huang T, Liu Y, Lei A, Ma C, Chen F and Chen M (2019) Effects of Attenuated S. agalactiae Strain

YM001 on Intestinal Microbiota of

Tilapia Are Recoverable.

Front. Microbiol. 9:3251.

doi: 10.3389/fmicb.2018.03251

\section{Effects of Attenuated S. agalactiae Strain YM001 on Intestinal Microbiota of Tilapia Are Recoverable}

\author{
Ming $\mathrm{Li}^{1+}$, Liping $\mathrm{Li}^{1+}$, Ting Huang ${ }^{1 \dagger}$, Yu Liu ${ }^{1}$, Aiying Lei ${ }^{1}$, Chunxia Ma ${ }^{2}$, Fuyan Chen ${ }^{1}$ and \\ Ming Chen ${ }^{1 *}$ \\ ${ }^{1}$ Guangxi Key Laboratory of Aquatic Genetic Breeding and Healthy Aquaculture, Guangxi Institute of Fisheries, Nanning, \\ China, ${ }^{2}$ Guangxi Key Laboratory of Animal Vaccines and Diagnostics, Department of Bacteriology, Guangxi Veterinary \\ Research Institute, Nanning, China
}

Previously, we constructed and characterized the vaccine efficacy of attenuated S. agalactiae strain YM001 in tilapia. In this study, the potential impacts of YM001 on the tilapia intestinal microbiota were assessed by qPCR and 16S rRNA sequencing methods. The results showed that YM001 distributed unevenly in different parts of intestine, peaked in the intestine at $12 \mathrm{~h}$ after oral administration, and then declined gradually. YM001 caused 0\% mortality of fish during the entire experimental period, while the referent strain HN016 caused 100\% mortality at $3 \mathrm{~d}$ after oral administration. However, the intestinal microbiota could be changed by YM001, the diversity of intestinal microbiota decreased first and gradually recovered after oral administration. The diversity of intestinal microbiota of tilapia was negatively correlated with the content of HN016 in the intestinal tract. The oral YM001 mainly changed the abundance of Streptococcus, Cetobacterium, Akkermansia, Romboutsia, Bacteroides, Brevinema, Lachnospiraceae_NK4A136-group, coprothermobactter, presiomonas, and Roseburia in intestine. The present study indicate that oral administration of YM001 altered the diversity and composition of intestinal microbiota in tilapia, but these change were only temporary, non-lethal, and recoverable. The results provide a more comprehensive experimental basis for the safety of oral YM001 vaccines.

Keywords: gut microbiota, oral vaccine, Streptococcus agalactiae, tilapia, microbiology

\section{INTRODUCTION}

Streptococcus agalactiae, or Group B streptococcus (GBS) is an important pathogen causing sepsis and meningitis in newborns and mastitis in bovine (Edwards and Baker, 2005; Tazi et al., 2011; Edmond et al., 2012; Lamagni et al., 2013). S. agalactiae is also a common aquaculture pathogen that can harm different fish species, such as tilapia, Barcoo grunter (Scortum barcoo), golden pompano (Trachinotus blochii), ya-fish (Schizothorax potanini), giant queensland grouper (Epinephelus lanceolatus), and silver pomfret (Pampus argenteus) (Jafar et al., 2008; Amal et al., 2012; Li et al., 2015). Tilapia is an important aquaculture species. Outbreaks of S. agalactiae in tilapia have cost more than 40 million dollars every year worldwide (Mian et al., 2009; Chen et al., 2012). Currently, S. agalactiae control in tilapia mainly relies on antibiotics, but the overuse of antibiotics will lead to food safety and problems with drug-resistant strains. Therefore, the use of antibiotics in aquaculture is severely restricted (Depaola et al., 1995; Baquero et al., 2008). 
Vaccines are the safest and most reliable method for the prevention of human and animal infectious diseases, but most of them are given by injection. However, in the field of aquatic products, the use of injections for immunization is greatly limited due to the large population of aquatic animals and the greater stress response resulted from the injection of vaccines. Compared to injectable vaccines, oral vaccines are more suitable for aquaculture animals. These vaccines are generally classified as live attenuated vaccines (LAVs), inactivated vaccines and subunit vaccines. Compared with inactivated vaccines or subunit vaccines, oral LAV have great advantages by avoiding the degradation of intestinal enzymes, directly entering the liver, kidney, spleen, and other immune related sites and simultaneously stimulating systemic immunity and local mucosal immunity (Chen et al., 2015; Makesh et al., 2015). Because of the above advantages, three types of LAVs against $S$. agalactiae in tilapia have been developed recently (Pridgeon and Klesius, 2013; Huang et al., 2014; Li et al., 2015).

Although oral LAVs have many advantages in application, their effects on the intestinal microbiota cannot be ignored. Long-term natural selection and differences in habitat, diet and habits have led to the formation of unique microbial symbiosis among different animals (Rawls et al., 2004; Ley et al., 2008). For fish, the micro-ecosystem composed of gut microorganisms is also formed during long-term historical evolution. These microorganisms are involved in the development of fish epithelial barrier, nutrition, digestion, as well as immunity (Gómez and Balcazar, 2008; Kelly, 2010; Roeselers et al., 2011; Sommer and Backhed, 2013; Xia et al., 2014). However, the balance of intestinal community composition could be altered by many factors (Xia et al., 2014), including stress (O'mahony et al., 2009; Kelly, 2010), antibiotic exposure (Tanaka et al., 2009), nutritional status (Turnbaugh et al., 2009), age (Hopkins et al., 2002), degree of hygiene (Schmidt et al., 2011), and bacterial infection (Dethlefsen et al., 2006). Gut microorganisms are continuously shaping the development of their host's immune system, directly modulating the innate and adaptive immune responses (Sommer and Backhed, 2013; Barroso-Batista et al., 2015). Differences in the host's intestinal microbiota can affect the effectiveness of the vaccine. For instance, intestinal microbiome composition correlates significantly with the immunogenicity of rotavirus vaccine (RVV) and may contribute to the diminished RVV immunogenicity. RVV response is correlated with an increased abundance of Streptococcus bovis and a decreased abundance of Bacteroidetes phylum (Harris et al., 2017). Therefore, it is necessary to analyze the effect of oral vaccines on intestinal microbiota.

In the previous study, we attenuated and domesticated the virulent $S$. agalactiae strain HN016 and obtained a safe, stable, and highly immunogenic attenuated S. agalactiae strain YM001. Oral immunization of tilapia with this strain produced a good immune protection (Chen et al., 2012; Li et al., 2015; Wang et al., 2015). Despite this, the effect of oral administration of YM001 on tilapia intestinal microbiota is still unknown. In this study, we analyzed the pathogenicity of oral administration of YM001 as well as its distribution in the intestine and influence on intestinal microbiota in tilapia using quantitative PCR and 16S bacterial sequencing with the hope to provide a more comprehensive experimental basis for the safety of YM001 oral vaccines.

\section{MATERIALS AND METHODS}

\section{Ethics Statement}

This study was carried out in accordance with the principles of good animal practice as defined by the European Union guidelines for the handling of laboratory animals (http://ec. europa.eu/environment/chemicals/lab_animals/home_en.htm) and the protocol was approved by Guangxi Institute of Fisheries Animal Ethics Committee and Guangxi Medical University Animal Ethics Committee. The institute did not issue a number or ID to this animal study, because the studied fish are not an endangered or protected species.

\section{Bacterial Strains and Fish}

S. agalactiae strain HN016 (serotype Ia) was isolated from an outbreak epidemical disease in tilapia from Hainan, China in 2010 (Chen et al., 2012). S. agalactiae strain YM001 was obtained by continuously passaging HN016 in vitro for 840 passages (Li et al., 2015). These two strains were stored at $-80^{\circ} \mathrm{C}$. Noninfected Nile tilapia was obtained from the National Tilapia Seed Farm (Nanning, Guangxi, China), with average weight of 155.45 $\pm 20.85 \mathrm{~g}$. Prior to experiments, the fishes were acclimated in plastic tanks ( $800 \mathrm{~L}$ each) with a stocking rate of $4 \mathrm{~g} / \mathrm{L}$ at $30 \pm$ $4^{\circ} \mathrm{C}$ for 2 weeks. The experimental fishes were checked randomly to verify pathogen free by bacteriological analysis of the brain and kidney samples.

\section{Oral Administration}

The bacteria for oral gavage were recovered and cultured as described previously ( $\mathrm{Li}$ et al., 2015). In brief, the stored HN016 and YM001 were thawed, streaked onto 5.0\% sheep blood agar plates, and incubated at incubator (Jing Hong, China) $28^{\circ} \mathrm{C}$ for $24 \mathrm{~h}$. Then single colony of every strain was inoculated in sterile tryptone soy broth (TSB) and incubated at $28^{\circ} \mathrm{C}$ for $24 \mathrm{~h}$ under low agitation. The concentration of the bacteria was determined as colony forming unit (CFU) per $\mathrm{mL}$ by plating $100 \mu \mathrm{L}$ of $10-$ fold serial dilutions onto blood agar plates. The details are as follow: $100 \mu \mathrm{L}$ culture of HN016 or YM001was added to $900 \mu \mathrm{L}$ PBS and blended. Then $100 \mu \mathrm{L}$ diluent was added to another 900 $\mu \mathrm{L}$ PBS and blended. By the same method, the culture was 10fold serial diluted to different concentration. Hundred micro liter diluent with different concentration were plated onto the blood agar plates and then incubated at $28^{\circ} \mathrm{C}$ for $24 \mathrm{~h}$. Only the agar plates with 30-100 colony of bacteria was selected to calculated the concentration of primary culture.

A total of 105 fishes were orally administered with HN016 or YM001 at the dose of $1.0 \times 10^{9} \mathrm{CFU} /$ fish (35 fishes/tank, with 3 replicates), and the control group was treated with $1.0 \mathrm{~mL} / \mathrm{fish}$ of TSB. The experimental fishes were cultured in plastic tanks $(800 \mathrm{~L}$ each) at $30 \pm 4^{\circ} \mathrm{C}$, then monitored and fed twice a day for 30 days. Five fishes in each group were sampled randomly at $0 \mathrm{~h}, 12 \mathrm{~h}$, $24 \mathrm{~h}, 3 \mathrm{~d}, 7 \mathrm{~d}, 15 \mathrm{~d}$ after oral gavage. Sampled fishes were dissected immediately with sterile scissors. The intestine was aseptically removed from their abdominal cavity, and cut into duodenum, 
foregut, midgut and hindgut, separately. All the samples then were stored at $-80^{\circ} \mathrm{C}$ immediately until use.

\section{DNA Extraction and Real-Time RT-PCR Assays}

Microbial DNA was extracted from fish intestine samples by using the Bacterial DNA Kit (50) (Takara, Japan) following the manufacturer's protocol. For each sample, DNA was extracted in triplicate to avoid bias, and the extracts from the same sample were pooled. The purity of DNA extracts was verified by electrophoresis on ethidium bromide staining $1 \%$ agarose gels, and their concentration was analyzed spectrophotometrically. To detect the distribution of YM001 or HN016 in tilapia intestines, specific primers and probe for $S$. agalactiae were design according to the $\mathrm{cfb}$ gene (Gene ID: 3686873). The Primer and probe sequences are listed in Table 1. Real-time PCR assays were performed using the Premix Ex Taq according to the protocol of Probe qPCR Kit (Takara, Japan) in a LightCycler 480 System (Roche, Germany). The PCR assays were performed at a volume of $20.0 \mu \mathrm{L}$ comprised of $10.0 \mu \mathrm{L}$ of SYBR Green I Master Mix (Takara, Japan), $0.5 \mu \mathrm{L}$ of cfb-F, $0.5 \mu \mathrm{L}$ of cfb-R, $0.5 \mu \mathrm{L}$ of TaqMan Probe, $4.0 \mu \mathrm{L}$ of template DNA and $7.5 \mu \mathrm{L}$ of $\mathrm{ddH}_{2} \mathrm{O}$. The cycling parameters were $30 \mathrm{~s}$ at $95^{\circ} \mathrm{C}$ followed by 40 cycles of $5 \mathrm{~s}$ at $95^{\circ} \mathrm{C} 5$ and $30 \mathrm{~s}$ at $60^{\circ} \mathrm{C}$. In blank control group, $\mathrm{ddH}_{2} \mathrm{O}$ was used to replace the template DNA. All Real-time PCR reactions were performed in triplicate.

\section{Illumina HiSeq Sequencing}

The extracted genomic DNA samples were diluted to $1 \mathrm{ng} / \mu \mathrm{l}$ with sterile water and used as templates to amplify the selected sequencing region using specific primers with barcodes and DNA polymerase with high efficiency and high fidelity. The PCR products were separated on $2 \%$ agarose gels, purified and prepared as libraries using TruSeq ${ }^{\circledR}$ DNA PCR Free Sample Preparation Kit. The constructed libraries were analyzed using Tapestation for quality control and Qubit and qPCR for quantification and sequenced using a Hiseq 2500PE250 platform.

The obtained FASTQ data were demultiplexed. After removal of barcodes and poor quality data, the obtained effective reads were analyzed using Upplese software (Uparse v7.0.1001, http:// drive5.com/uparse/) and clustered into operational taxonomic units (OTUs) based on 97\% identity. The sequences of each OUT with the highest abundance were selected as the representative sequence and used for annotation against

TABLE 1 | Sequences of primers and probe.

\begin{tabular}{ll}
\hline Primer & Sequence $\mathbf{( 5}^{\prime}$ to $\left.\mathbf{3}^{\prime}\right)$ \\
\hline $\mathrm{cfb}-\mathrm{F}$ & CGGTTAATGAGGCTATTACTAGTG \\
$\mathrm{cfb}-\mathrm{R}$ & ATCTGTTAAGGCTTCTACACGAC \\
$\mathrm{cfb}-P$ probe & FAM-TTCATTGCGTGCCAACCCTGAGACA-EClipse
\end{tabular}

the GreenGene database (http://greengenes.secondgenome.com) using the RDP Classifier (Version 2.2, http://sourceforge.net/ projects/rdp-classifier/) at the setting threshold of $0.8-1$ to calculate the community composition of each sample at the levels of kingdom, phylum, class, order, family, genus, and species.

The data for each sample was normalized and subjected to Alpha diversity analysis and Beta diversity analysis.

\section{RESULTS}

\section{Disease Symptoms of the Experimental Fish}

All fish in the YM001 group showed no significant changes except that their appetite dropped at $24-72 \mathrm{~h}$ after oral administration of YM001. The appetite returned to normal at $4 \mathrm{~d}$ after the administration. No fish in the YM001 group died during the entire experiment period. By contrast, fish in the HN016 group showed typical symptoms of $S$. agalactiae infection at $24 \mathrm{~h}$ after administration of HN016 including lack of energy, abnormal swimming posture and body blackness and died $3 \mathrm{~d}$ after administration of HN016.

\section{Distribution of YM001 in Fish Intestine}

Tilapia intestinal samples were collected at $0 \mathrm{~h}, 12 \mathrm{~h}, 24 \mathrm{~h}, 3 \mathrm{~d}, 7$ $\mathrm{d}$, and $15 \mathrm{~d}$ after oral administration of $S$. agalactiae, respectively. Their DNA were extracted and analyzed using fluorescence quantitative PCR to detect the distribution and colonization of YM001 and HN016 in the intestine of tilapia. The results showed that both YM001 and HN016 can be colonized in the foregut, rectum, duodenum and hindgut, but their number and distribution were significantly different. The contents of YM001 and HN016 were the highest in all intestinal segments at $12 \mathrm{~h}$ and gradually decreased with time (Figures 1A,B). The relative reductions of YM001 and HN016 in different segments of the intestine were different. The reduction of YM001 was in the order of foregut $>$ rectum $>$ duodenum $>$ hindgut, and hindgut $>$ duodenum $>$ rectum $>$ foregut at $24 \mathrm{~h}-3 \mathrm{~d}$ (Figure 1C) while that of HN016 was duodenum $>$ foregut $>$ hindgut $>$ rectum (Figure 1D).

\section{$16 S$ rRNA Gene Sequencing Analysis of Intestinal Microbiota in the Experimental Tilapia}

The V3-V4 region of the 16S rRNA gene of each sample was sequenced on the Illumina HiSeq platform. A total of 2,891,942 original sequences and 2201874 effective reads were obtained from 36 samples. The total effective rate was $76.13 \%$. The number of original sequences per sample was 63,84292,065 and the effective read number was 48,541-70,400. The average length (AvgLen) was 405-426 nt (Table 2). To study the compositional diversity information of the microbiota, the effective sequences of all samples were clustered using the Uparse software into Operational Taxonomic Units (OTUs) according to $97 \%$ sequence similarity. A total of 964 OTUs were obtained from 36 tilapia intestinal samples with average OTUs of 339-1,623 per sample. 

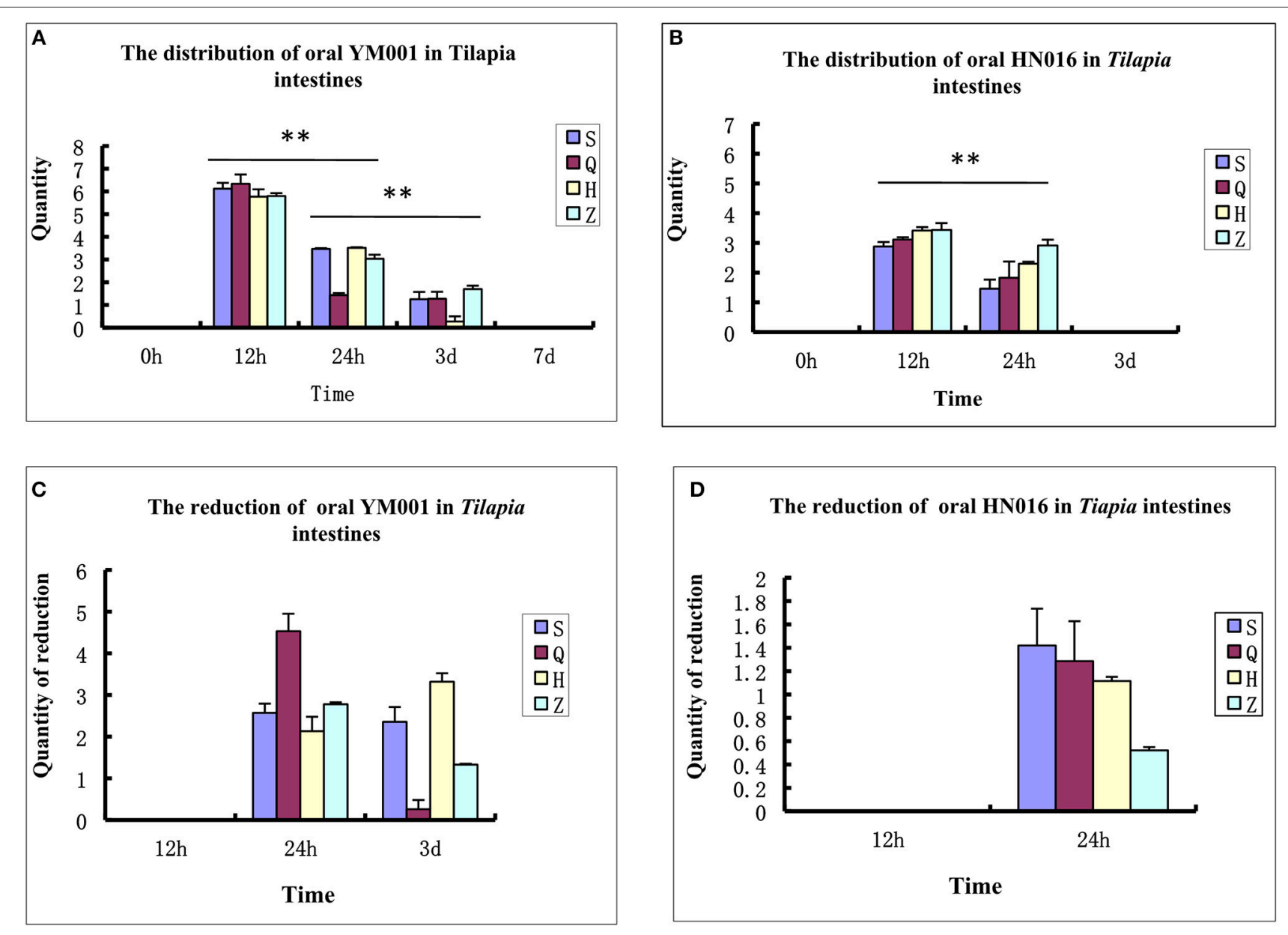

FIGURE 1 | The retention time of YM001 and HN016 is different in the intestinal tract. (A) The distribution of YM001 in Tilapia intestines. (B) The distribution of HNO16 in Tilapia intestines. (C) The reduction of YM001 in Tilapia intestines. (D) The reduction of HN016 in Tilapia intestines. Real-time RT-PCR was performed in triplicate for each sample. The data were provided as the mean fold changes (means \pm S.D., $n=3$ ) relative to the control group. The statistical significance was calculated by the Student's $t$-test $\left({ }^{* *} p<0.01\right)$.

\section{Distribution of Microorganisms in Various Intestinal Segments of Tilapia Before Oral Administration of S. agalactiae}

Analysis of the Venn diagram of intestinal microbiota showed that 1,359 OTUs were shared in the intestine of normal tilapia, and the number of OTUs unique to the duodenum, foregut, hindgut, rectum was 415, 365, 364, and 469, respectively (Figure 2A).

At the phylum level, the dominant microbial floras in tilapia intestine are mainly firmicutes, fusobacterium, proteobacteria, bacteroidetes, actinobacteria, thaumarchaeota, chloroflexi, tenericutes, verrucomicrobia, synergistetes, and actidobacteria (Figure 2C). The dominant microbiota in tilapia duodenum were proteobacteria (22.14.38\%), bacteroidetes (18.02\%), fusobacterium (17.41\%), firmicutes (12.39\%), actinobacteria (4.77\%), chloroflexi (1.79\%), and Others (23.37\%); The dominant microbiota in tilapia foregut were Firmicutes (68.67\%), fusobacterium (12.12\%), proteobacteria (8.99\%), bacteroidetes (4.86\%), actinobacteria (4.23\%), and others (1.12\%); The dominant microbiota in tilapia hindgut were Firmicutes $(36.66 \%)$, proteobacteria $(28.41 \%)$, bacteroidetes (18.56\%), fusobacterium (6.84\%), actinobacteria (4.68\%), Chloroflexi (1.79\%), Others (23.37\%); The dominant microbiota in tilapia rectum were fusobacterium (29.96\%), firmicutes (18.86\%), proteobacteria (11.35\%), bacteroidetes $(4.32 \%)$, actinobacteria (4.08\%), thaumarchaeota (2.99\%), tenericutes (1.29\%), and others (27.14\%).

At genus level, different positions of tilapia intestine also have different dominant bacterial structures (Figure 2D). The dominant microbiota in tilapia duodenum were streptococcus, sphingomonas, rhodanobacter, arthrobacter, tumebacillus, pedobacter, vadinBC27_wastewater-sludge_group; paenibacillus and anaerobaculum; the dominant microbiota in tilapia foregut were romboutsia, blautia, propionibacterium, pseudobutyrivibrio, ruminiclostrid_9, allobaculum, peptoniphilus, lactococcus, prevotella and micrococcus; the 
TABLE 2 | Statistics the data of the samples sequencing.

\begin{tabular}{|c|c|c|c|c|}
\hline ID & $\begin{array}{c}\text { Raw reads } \\
\text { number }\end{array}$ & $\begin{array}{l}\text { Effective } \\
\text { reads number }\end{array}$ & $\begin{array}{c}\text { Effective reads } \\
\text { ratio }(\%)\end{array}$ & AvgLen (bp) \\
\hline S1 & 67,202 & 51,024 & 75.93 & 407 \\
\hline Q1 & 87,705 & 63,545 & 72.45 & 415 \\
\hline $\mathrm{H} 1$ & 88,978 & 63,048 & 70.86 & 418 \\
\hline Z1 & 80,330 & 61,860 & 77.00 & 400 \\
\hline RS1 & 85,012 & 66,061 & 77.71 & 411 \\
\hline RQ1 & 69,790 & 53,911 & 77.25 & 399 \\
\hline $\mathrm{RH} 1$ & 77,753 & 60,507 & 77.82 & 411 \\
\hline RZ1 & 87,127 & 68,186 & 78.26 & 414 \\
\hline RS2 & 82,023 & 60,768 & 74.09 & 423 \\
\hline RQ2 & 77,184 & 56,278 & 72.91 & 425 \\
\hline $\mathrm{RH} 2$ & 90,328 & 67,106 & 74.29 & 425 \\
\hline RZ2 & 92,065 & 68,313 & 74.20 & 424 \\
\hline RS3 & 79,045 & 60,180 & 76.13 & 414 \\
\hline RQ3 & 80,394 & 62,708 & 78.00 & 408 \\
\hline $\mathrm{RH} 3$ & 76,219 & 59,523 & 78.09 & 413 \\
\hline RZ3 & 88,974 & 68,382 & 76.86 & 411 \\
\hline RS4 & 81,896 & 61,065 & 74.56 & 413 \\
\hline RQ4 & 84,224 & 67,639 & 80.31 & 412 \\
\hline $\mathrm{RH} 4$ & 75,255 & 58,985 & 78.38 & 410 \\
\hline RZ4 & 80,641 & 64,536 & 80.03 & 413 \\
\hline RS5 & 64,970 & 48,541 & 74.72 & 404 \\
\hline RQ5 & 80,847 & 63,449 & 78.48 & 407 \\
\hline $\mathrm{RH} 5$ & 75,724 & 60,644 & 80.09 & 407 \\
\hline RZ5 & 89,025 & 70,400 & 79.08 & 409 \\
\hline RS6 & 83,729 & 60,600 & 72.38 & 409 \\
\hline RQ6 & 63,842 & 50,847 & 79.65 & 403 \\
\hline $\mathrm{RH} 6$ & 81,714 & 59,235 & 72.49 & 407 \\
\hline RZ6 & 71,835 & 55,227 & 76.88 & 412 \\
\hline QS1 & 90,604 & 67,067 & 74.02 & 422 \\
\hline QQ1 & 86,118 & 65,332 & 75.86 & 416 \\
\hline QH1 & 69,187 & 52,006 & 75.17 & 415 \\
\hline QZ1 & 76,567 & 58,042 & 75.81 & 419 \\
\hline QS2 & 82,843 & 58,386 & 70.48 & 426 \\
\hline QQ2 & 82,196 & 63,483 & 77.23 & 412 \\
\hline $\mathrm{QH} 2$ & 77,184 & 60,891 & 78.89 & 425 \\
\hline QZ2 & 83,312 & 64,101 & 76.94 & 418 \\
\hline
\end{tabular}

dominant microbiota in tilapia hindgut were Acinetobacter, Lactobacillus, Lachnospiraceae_NK4A136_group, Rubrobacter, Sporolactobacillus, Bacteroides Alloprevotella, Stenotrophomonas and Prevotella_9; and the dominant microbiota in tilapia rectum were Anaerobaculum, Ruminococcaceae_UCG-014, Ruminococcus_2, Acetitomaculum, Candidatus_Nitrosopumilus, Lachnospiraceae_NK3A20_group, Streptomyces, Cetobacterium.

The clustering tree analysis showed that the microbiota in tilapia foregut and hindgut were more similar than that in the duodenum and rectum (Figure 2E). The dilution curve can directly reflect the rationality of the sequencing data and indirectly reflect the abundance of species in the sample. Our results in the dilution curves showed that the diversity of microbes in different gut segments of tilapia was different, showing an order of rectum $>$ duodenum $>$ hindgut $>$ foregut in species richness (Figure 2B).

\section{Effect of YM001 on Intestinal Microbiota in Tilapia}

$\beta$ diversity analysis of intestinal samples of tilapia found that the diversity of gut microbiota lowered after oral administration of YM001, reaching the lowest at $12 \mathrm{~h}$ and gradually recovered afterward to the normal level at 15 d (Figure 3). Furthermore, YM001 significantly changed the composition of microbiota in tilapia intestine, and the relative proportion of dominant bacteria fluctuated at different time (Figure 4A). At genus level, the top 10 intestinal microbe were Streptococcus, Cetobacterium, Akkermansia, Romboutsia, Brevinema, Lachnospiraceae_NK4A136-group, Coprothermobactter, Plesiomonas, and Roseburia after the YM001 administration. The abundance of Streptococcus in the intestine reached the highest level (70.8\%) at $12 \mathrm{~h}$ after YM001 administration, then dramatically decreased at $24 \mathrm{~h}$ after YM001 administration, and almost restored to the normal level at $5 \mathrm{~d}$ and $7 \mathrm{~d}$ after YM001 administration (Table 3). The trend of changes in Streptococcus abundance is consistent with that measured by fluorescence quantitative PCR (Figure 1). Cetobacterium accounted for approximately $25 \%$ of the total bacteria before oral administration of YM001 and showed a significant decrease at $12 \mathrm{~h}$ after administration. After that, the abundance of Cetobacterium rebounded to $25 \%$ at $24 \mathrm{~h}$, decreased again at 3 $\mathrm{d}$ after administration and almost returned to the original level at 7 and $15 \mathrm{~d}$ after administration (Table 3). The abundance of Bacteroides was about $2.2 \%$ before oral administration of YM001 but increased to $12.4 \%$ at $24 \mathrm{~h}$ after administration and then gradually decreased to the original level later (Table 3). Akkermansia accounted for about $1 \%$ of the total bacteria before oral administration of YM001, but the abundance rose to $28.3 \%$ at $3 \mathrm{~d}$ after oral administration of YM001 and then gradually decreased at 5 and $7 \mathrm{~d}$ (Table 3). Romboutsia accounted for about $1.5 \%$ of the total bacteria before oral administration of YM001, but the abundance reduced to $0.8 \%$ at $12 \mathrm{~h}$ after oral administration of YM001 and then gradually increased to $17.7 \%$ at $15 \mathrm{~d}$ (Table 3). The content of Brevinema increased to $7 \%$ at $15 \mathrm{~d}$ after oral administration of YM001, and was less than $1 \%$ at all other times (Table 3).

The contents of Lachnospiraceae_NK4A136-group, Coprothermobactter, Plesiomonas, and Roseburia in the intestine also fluctuated but to less extent compared with the other genera. Before oral administration of YM001, the content of Lachnospiraceae_NK4A136-group was 7\% in duodenum and $<1 \%$ in other segments, after oral administration of YM001, its content showed a decreasing trend (Table 3). The content of Presiomonas was approximately $0.4 \%$ in total intestinal bacteria in the case of normal, but after oral administration of YM001, its content rose to $2.4 \%$ at $12 \mathrm{~h}$ and $3.9 \%$ at $24 \mathrm{~h}$, and then gradually reduced to $1.3 \%$ at $15 \mathrm{~d}$, close to the initial level (Table 3). Before oral administration of YM001, the content of Coprothermobactter in each gut segment was 


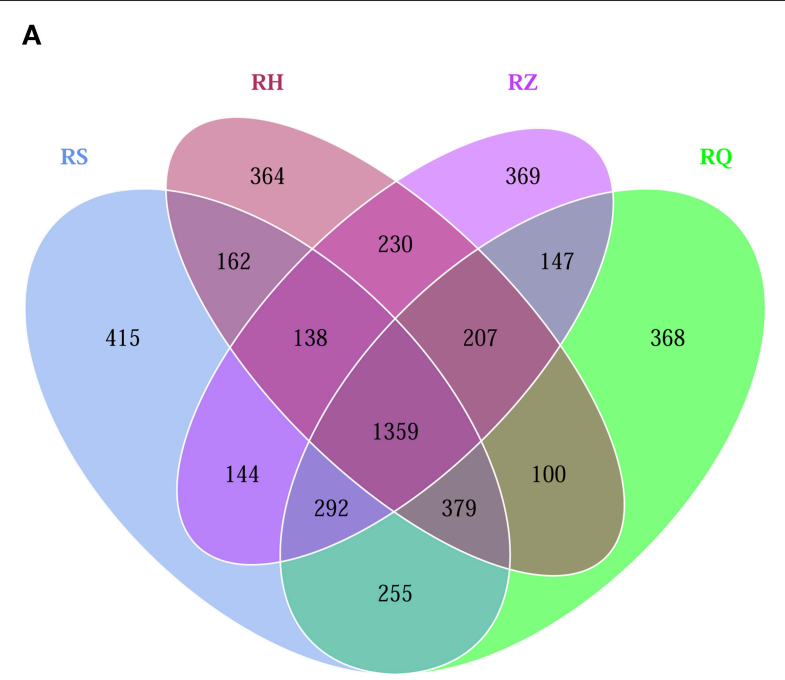

D

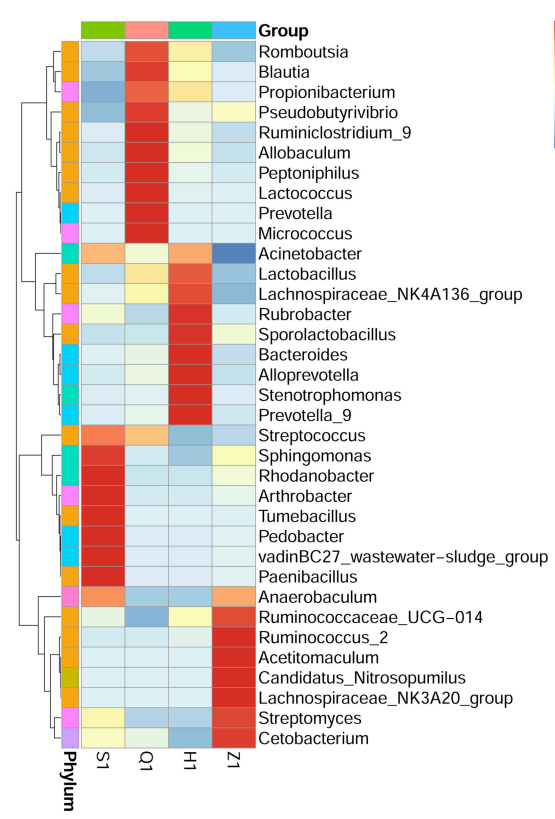

B

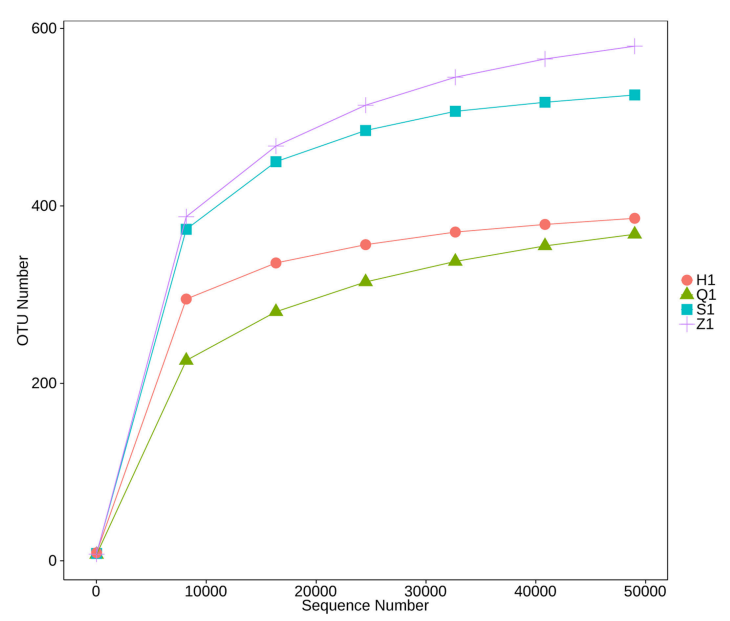

C

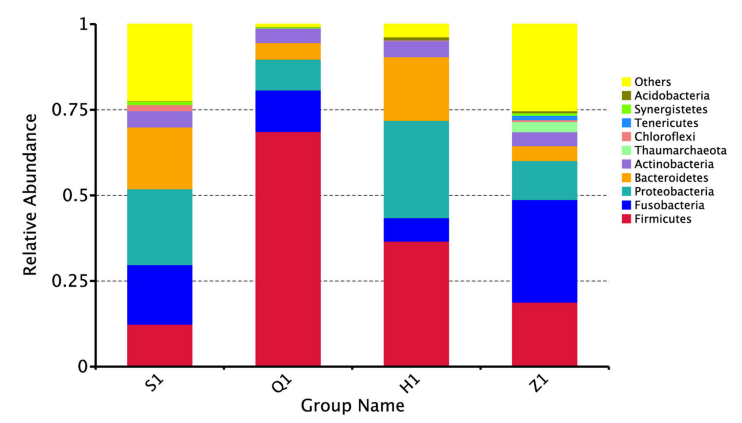

E

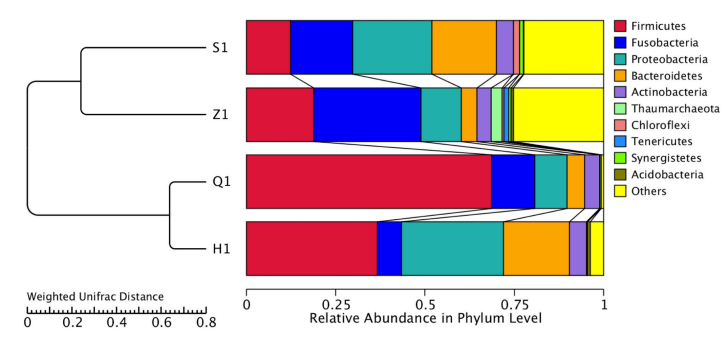

FIGURE 2 | The intestinal flora in different segments of tilapia were different in structure and diversity. (A) Venn diagram (Petals figure) illustrating the Common or characteristic OTUs in duodenum (S), foregut $(\mathrm{Q})$, midgut $(\mathrm{Z})$, hindgut $(\mathrm{H})$. (B) Rarefaction curves of OTUs illustrating the microbial diversity in duodenum (S), foregut $(Q)$, midgut $(Z)$, hindgut $(H)$. When the curve flattens, it shows that the amount of sequencing data is gradually reasonable, and more data will produce only a small number of new species (OTUs). (C) Column diagram illustrating the relative abundance of Intestinal flora at phylum level in duodenum (S), foregut (Q), midgut (Z), hindgut $(\mathrm{H})$. The horizontal axis is the sample name, and the vertical axis represents the relative abundance of a classification. Different colors correspond to different species at the same level. (D) Heat maps illustrating the relative abundance of Intestinal flora at genus level in duodenum (S), foregut (Q), midgut (Z), hindgut (H). The color and intensity of each square represent the value of relative abundance. (E) UPGMA clustering tree illustrating the similarity between samples of duodenum (S), foregut $(Q)$, midgut $(Z)$, hindgut $(H)$.

$<0.1 \%$. However, at $3 \mathrm{~d}$ after administration of YM001, its content in the foregut increased to $7 \%$ and then returned to the normal level at 7 and $15 \mathrm{~d}$ after administration of YM001 (Table 3). Before oral administration of YM001, the content of Roseburia in each gut segment was $<0.1 \%$ except that of $6.3 \%$ in duodenum. After administration, its content in duodenum gradually decreased, reaching to the normal level at 7 and $15 \mathrm{~d}$ after administration of YM001 (Table 3).

In addition to the above-mentioned predominant bacteria, oral administration of YM001 also caused changes in other nonpredominant gut bacteria. The heat maps show the changes in 

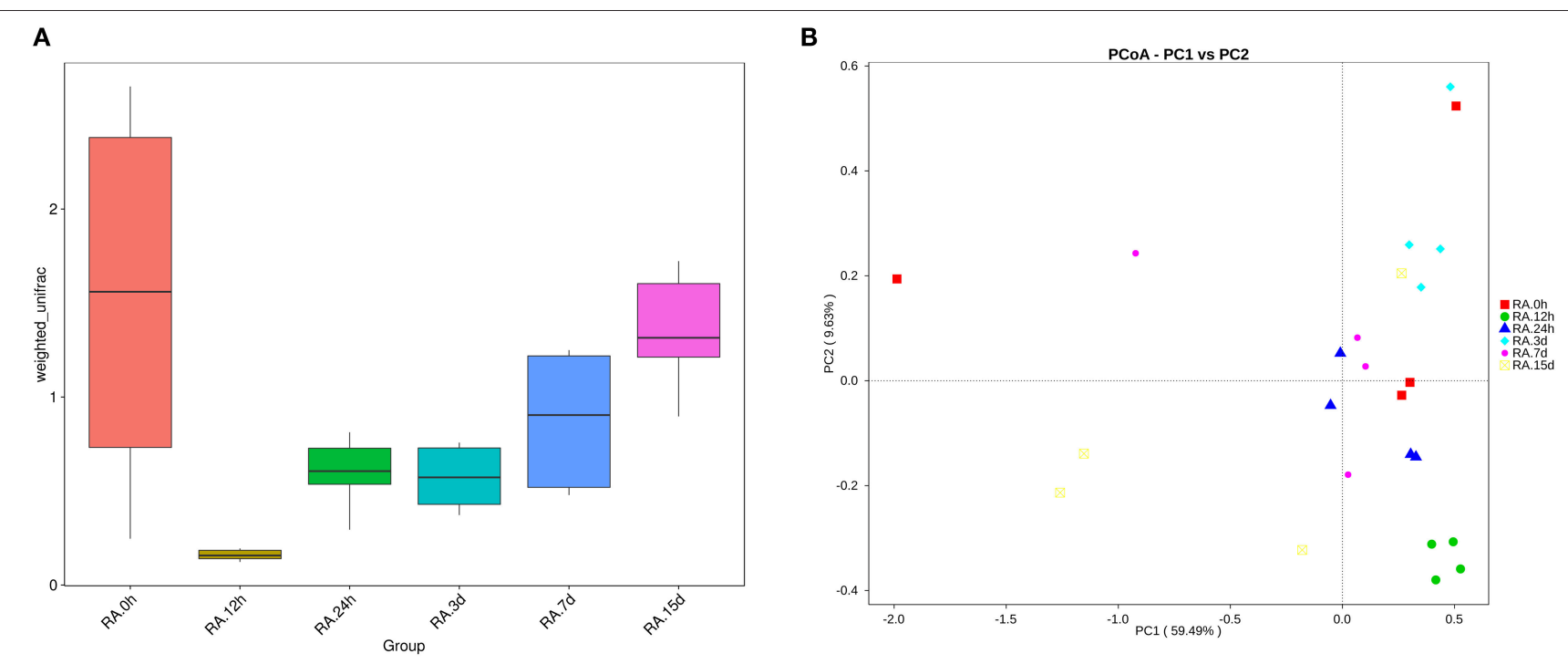

FIGURE 3 | YM001 alters the diversity of intestinal flora of tilapia at different observing times. (A) A box chart based on Weighted Unifrac Beta diversity. (B) Principle coordinate analysis (PCoA) Based on Weighted Unifrac distance. The samples at $0 \mathrm{~h}, 12 \mathrm{~h}, 24 \mathrm{~h}, 3 \mathrm{~d}, 7 \mathrm{~d}$, $15 \mathrm{~d}$ were represented as color icons with red square, green dot, blue triangle, turquoise square, pink dot, orange box.

the highest 35 gut bacteria at both phylum and genus levels (Figures 4B,C).

\section{Differences of Gut Microbial Changes in Tilapia Subjected to Oral Administration of YM001 and HN016}

Anosim analysis is a non-parametric test used to examine whether the differences between groups are significantly greater than the differences within groups. Comparison of intestinal microbiota composition between any two group, YM001 and HN016 or normal control group $(0 \mathrm{~h})$,showed that all the Rvalues of Anosim analysis were $>0$ (Figures 5A-D), indicating that the composition of intestinal microbiota between any two group was significantly different and the difference between the groups were greater than that within the group.

To determine whether two different $S$. agalactiae strain altered the composition of the gut microbiota in a similar pattern, we compared the diversity and richness indices of intestinal microbiota of tilapia at different periods after administration of YM001 and HN016. The results showed that both Chao1 index and Shannon index dropped at $12 \mathrm{~h}$ after administration of YM001 or HN016, then increased again at $24 \mathrm{~h}$. Especially, the Chaol index of YM001 or HN016 groups at $24 \mathrm{~h}$ was higher than that of the control groups. Except the Chaol index at $12 \mathrm{~h}$, the Chaol index and the Shannon index of YM001 groups at all other time points were lower than those of HN016 groups (Figures 6A,B). These data indicated that the richness of the intestinal microbiota of tilapia at $12 \mathrm{~h}$ after administration of YM001 or HN016 were attenuated, especially in tilapia after $12 \mathrm{~h}$ after oral administration of YM001.

LDA effect size (LEfSe) analysis is used to identify biomarkers or the species that have statistically significant differences between two groups. Comparing with other groups, the following biomarkers, including $k$ _Bacteria, p_Firmicutes, $c \_$_Bacilli, o__Lactobacillales, f_Streptococcaceae, $g$ __Streptococcus and s_Streptococcus_agalactiae, of YM001 group at $12 \mathrm{~h}$ were most different (Figures 7A,B). On other hand, the following biomarkers, including $p_{\text {_F Fusobacteria, }}$ c_Fusobacteriia, o_Fusobacteriales, f_Fusobacteriaceae, g_Cetobacterium, of YM001 group at $24 \mathrm{~h}$ were most different compared with other groups. In addition, the biomarkers $c \_$_Clostridia, o_CClostridiales, o_Pseudomonadales, o__Bacillales, f_Clostridiaceae_1, f_Pseudomonadaceae,

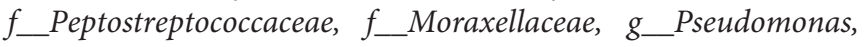
g_Plesiomonas, g_Romboutsia, g_Acinetobacter, g_Clostridium_sensu_stricto,s_Acinetobacter_baumannii, s_Pseudomonas_plecoglossicida were significantly different in the intestine of tilapia at $12 \mathrm{~h}$ after administration of subjected to virulent $S$. agalactiae strain HN016 compared with other groups; and the biomarkers $k$ __Archaea, $\quad$ __Tenericutes, $\quad p \_$Synergistetes, p_Euryarchaeota, p__Proteobacteria, c_Alphaproteobacteria, c_Methanomicrobia, $\quad c \_$Mollicutes, $\quad c$ _Synergistia c__Deltaproteobacteria, o__Synergistales, o__Desulfovibrionales, $f$ _Desulfovibrionaceae, $f$ _Synergistaceae, g_Anaerobaculum, and s_Acinetobacter_junii were significantly different in the intestine of tilapia at $24 \mathrm{~h}$ after administration of subjected to HN016 compared with other groups (Figures 7A,B).

\section{DISCUSSION}

For oral attenuated vaccines, safety and efficacy are critical. In this study, each tilapia was given $10^{9}$ YM001 or $10^{9}$ HN016 via oral gavage, respectively. Compared with $100 \%$ mortality of fish given HN016 within 3 d, the mortality of fish given YM001 


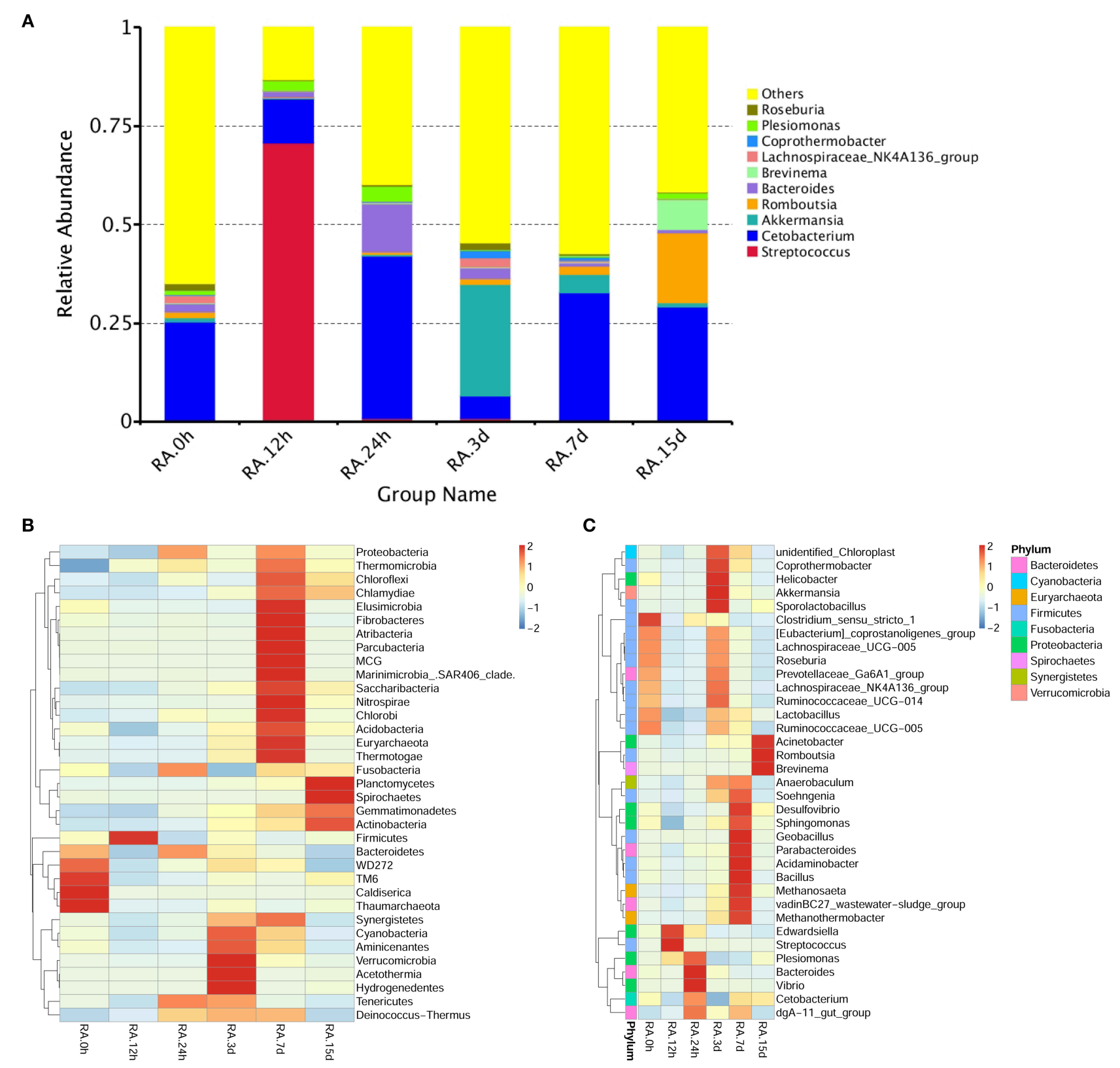

FIGURE 4 | YM001 alters the intestinal flora composition at different observing times. (A) The relative abundances of the top 10 bacteria at the genus level in YM001 groups. (B) The alteration of intestinal bacterial patterns at the genus level. The heat map is color-based on row Z-scores. The highest and lowest bacterial levels are showed in red and blue, respectively. (C) The alteration of intestinal bacterial patterns at the phylum level. The heat map is color-based on row Z-scores. The highest and lowest bacterial levels are showed in red and blue, respectively.

was $0 \%$ within $15 \mathrm{~d}$, indicating that YM001 has higher safety. The decrease of virulence of YM001 was related to the change of its genetic structure. Our previous data have shown that compared to HN016, YM001 lost two 5832bp, and 11,116 bp DNA fragments and possesses more than 40 genes with deletions or point mutations (Wang et al., 2015).

Live vaccines administrated via gavage in the intestine could stimulate immunity through two routes. On the one hand, live bacteria will colonize on the mucosa of intestine, stimulating mucosal immunity. On the other hand, these bacteria will break through the intestinal barrier and enter other immune organs, stimulating systemic immunity. Therefore, the number of colonization in the intestine, retention time, and intestinal barrier breakthrough efficiency of YM001 are related to the immune efficacy of the YM001 oral vaccine. In this study, realtime quantitative PCR was used to detect the retention of YM001 and HN016 in the intestine of tilapia. It was found that the content of both was the highest at $12 \mathrm{~h}$ after administration and 
TABLE 3 | The composition of the top ten intestinal flora in YM001 group.

\begin{tabular}{|c|c|c|c|c|c|c|c|c|c|c|c|}
\hline Taxonomy & Strep & Cetob & Akker & Romb & Bacte & Brevi & Lachn & Copro & Plesi & Roseb & Others \\
\hline RA.Oh & 0.005012 & 0.247655 & 0.01045 & 0.015298 & 0.022154 & 0.001102 & 0.019525 & 0.001347 & 0.011202 & 0.017022 & 0.649234 \\
\hline RA.12h & 0.708163 & 0.110283 & 0.002242 & 0.002841 & 0.014807 & 0.000169 & 0.001974 & 0.00042 & 0.024902 & 0.001407 & 0.132791 \\
\hline RA.24h & 0.008764 & 0.411216 & 0.003523 & 0.006643 & 0.124136 & 0.000976 & 0.003 & 0.000807 & 0.039513 & 0.002274 & 0.399147 \\
\hline RA.3d & 0.008623 & 0.056982 & 0.283192 & 0.014076 & 0.028616 & 0.000774 & 0.024422 & 0.018505 & 0.001767 & 0.016662 & 0.54638 \\
\hline RA.7d & 0.004887 & 0.322307 & 0.046462 & 0.020419 & 0.009822 & 0.000862 & 0.005208 & 0.007788 & 0.004058 & 0.004483 & 0.573704 \\
\hline RA.15d & 0.001756 & 0.290315 & 0.009332 & 0.177731 & 0.00895 & 0.076065 & 0.002476 & 0.00018 & 0.013984 & 0.001189 & 0.418023 \\
\hline
\end{tabular}

Strep, Streptococcusl; Cetob, Cetobacterium; Akker, Akkermansia; Romb, Romboutsia; Bacte, Bacteroides; Brevi, Brevinema; Lachn, Lachnospiraceae_NK4A136_group; Copro, Coprothermobacter; Plesi, Plesiomonas; Roseb, Roseburia.

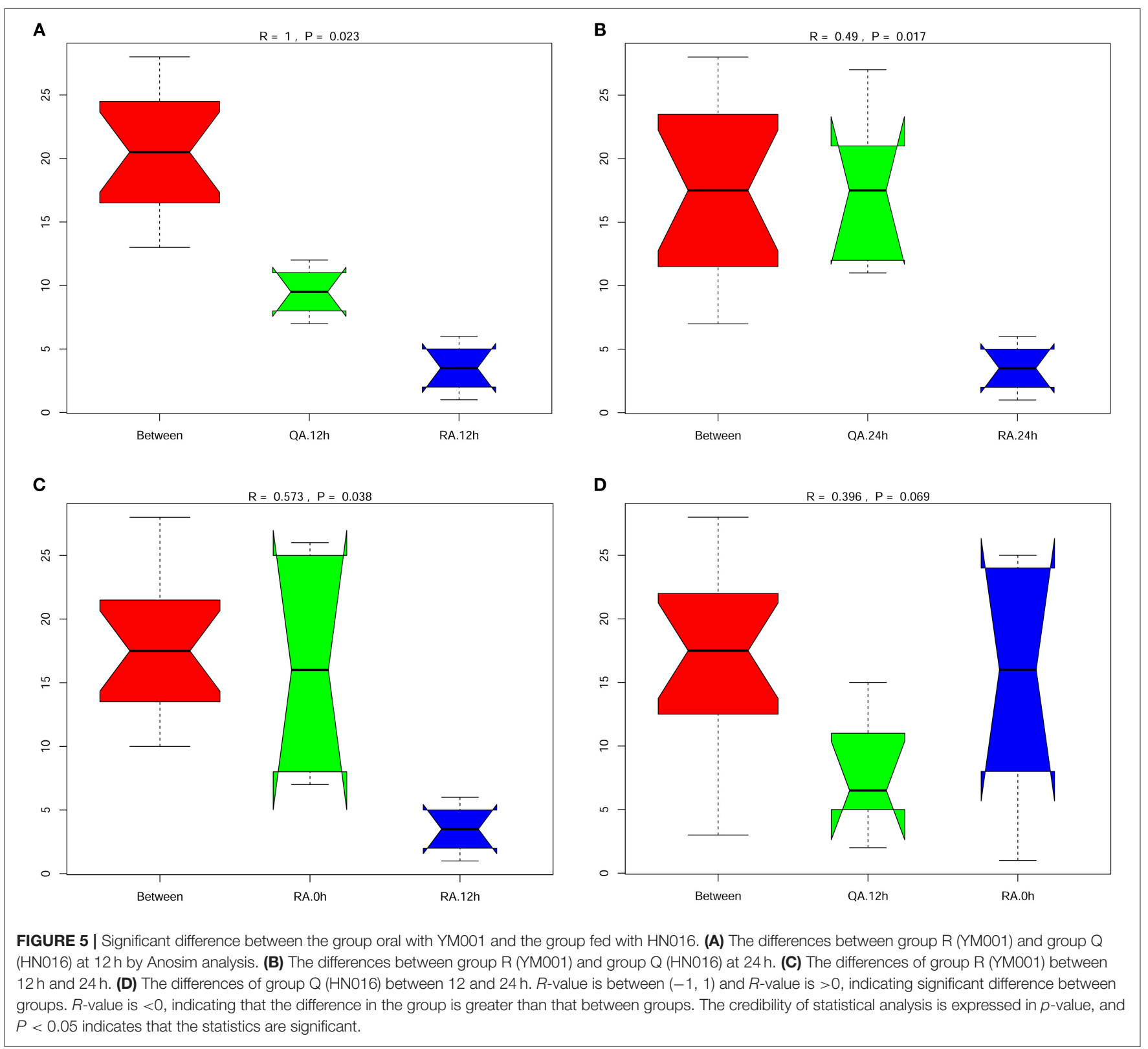



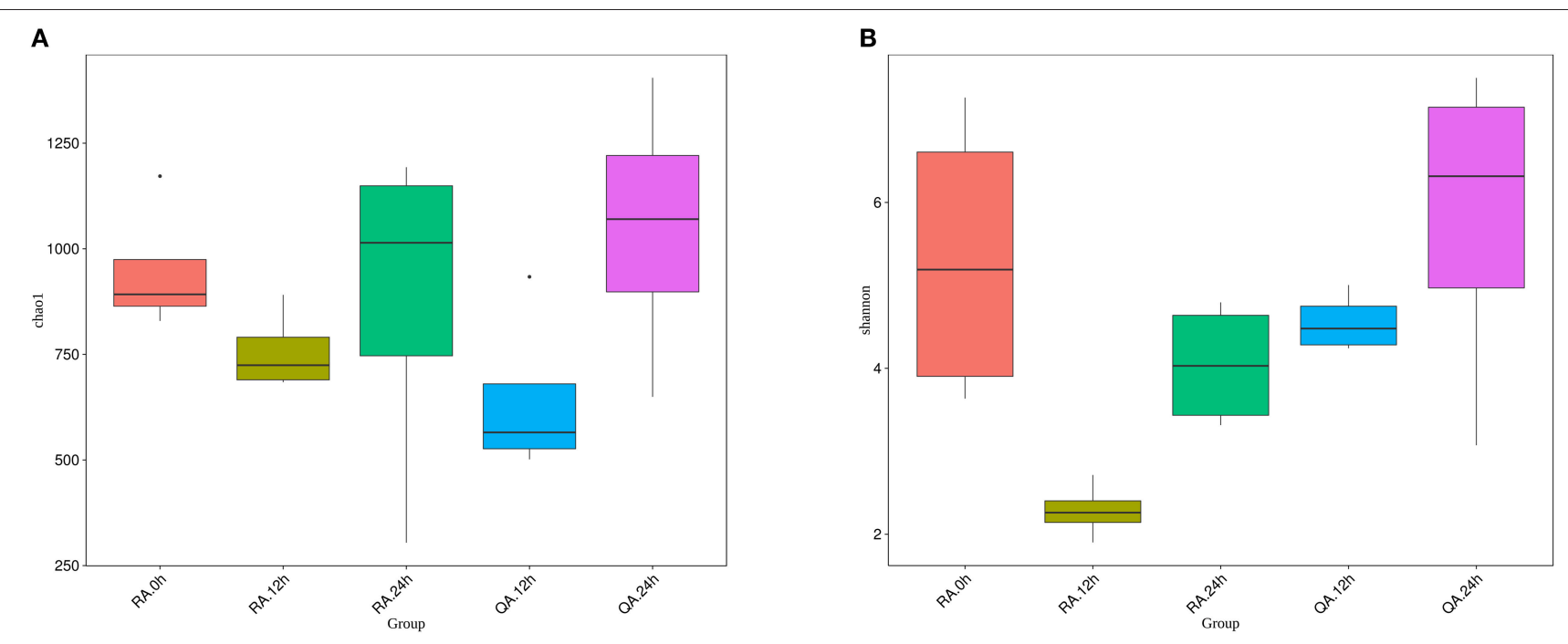

FIGURE 6 | The diversity of intestinal flora of tilapia, which fed with YM001 or HN016, changed differently. (A) Diversity of bacterial species as indicated by Chao1 rarefaction measure. The larger the number of OTU in the sample is, the larger the number of species in the sample. (B) Shannon index. The richness and evenness of the species composition in the sample were evaluated. The larger the value was, the more abundant the species was, and the more homogeneous the species were. Evenness based on species-level operational taxonomic units (OTUs). Error bars indicate $\mathrm{SD}$, ${ }^{*}$ indicates significant association $(0.005<P<0.05)$.

then greatly reduced to almost undetectable at 3d (HN016) or 7d (YM001) after administration (Figure 1A). The above results show that the intestinal environment of tilapia is not suitable for long-term colonization of YM001. The intestine as well as its intrinsic microbiota has a strong repulsive effect on YM001.

The symbiotic bacteria colonize in the intestinal mucosa of fish to form a bacterial plexus, which acts as a barrier against the colonization and proliferation of pathogens and to a certain extent protects the host against pathogens (Gómez and Balcazar, 2008; Kelly, 2010). Our previous studies showed that YM001 could be detected in the brain, liver, spleen, and kidney of tilapia at $6 \mathrm{~h}$ after administration of YIM001, and the levels of YM001 in the tissues gradually decreased after 24h of YM001 administration (Li et al., 2015), indicating that although YM001 is repulsed by the intestine or intrinsic microbiota of tilapia, it can still quickly cross the intestinal barrier to reach other parts of the body. The content of YM001 in the intestine and other organs began to decrease simultaneously at $24 \mathrm{~h}$ after oral administration, indicating that the defense responses of intestine in tilapia are highly synchronized with the internal defense responses of the body. The responses of tilapia intestine to YM001 and HN016 may be different. Comparison of the contents of YM001 and HN016 in various segments of the intestine showed that there was still a large amount of YM001 in tilapia intestine at $3 \mathrm{~d}$ after oral administration, but HN016 was almost undetectable, indicating that YM001 was likely to colonize on the intestine much longer that than HN016 (Figures 1A,B). In addition, comparison of the content of YM001 and HN016 in each segment of the intestine also showed that the concentration of YM001 was higher than that of HN016 at each time point except that the concentration of YM001 was lower than that of HN016 in foregut at $24 \mathrm{~h}$ after administration (Figures 1A-D).
There may be two reasons for this situation. One is that the virulent strain HN016 enters the intestinal barrier faster than the attenuated strain YM001, resulting in a lower content of HN016 in the intestine than that of the attenuated strain YM001. The other one is because HN016 could produce toxins, resulting in strong intestine responses, which accelerate the discharge of HN016, and eventually result in lower content in the intestine than that of the attenuated strain YM001. Regardless of the situation, the longer colonization time of the weak strain YM001 in the intestine is obviously beneficial to stimulate the body to produce long-lasting immunity.

To analyze the influence of YM001 on intestinal microbiota of tilapia, it is necessary to understand the structure of the intestinal microbiota of healthy tilapia. 16S RNA sequencing analysis showed that proteobacteria, firmicutes, fusobacterium, bacteroidetes, verrucomicrobia, spirochaetes, Euryarchaeota, actinobacteria, synergistetes, and tenericutes are the top abundant bacteria genera in the intestine of healthy tilapia (Figure 2C). Swapnil Sopan Gaikwad et al. used 16S rRNA gene sequencing on the Illumina Miseq platform and found that Fusobacteria and Proteobacteria are the dominated genera in the digestive tract of tilapia collected from rivers while Actinobacteria, Cyanobacteria, Planctomyctes, and Proteobacteria are the dominant genera in in the digestive tract of tilapia collected from lakes (Gaikwad et al., 2017). Our results are similar to those of Swapnil Sopan Gaikwad et al., but there are also some bacterial species that are inconsistent with their research, such as Verrucomicrobia, Spirochaetes, Euryarchaeota, Synergistetes, and Tenericutes. These discrepancies may be related to the experimental fish species, different living environments and diet, and may also be related to different sequencing methods and sequencing depths. Microbial colonies are widely distributed in the digestive tract and are 


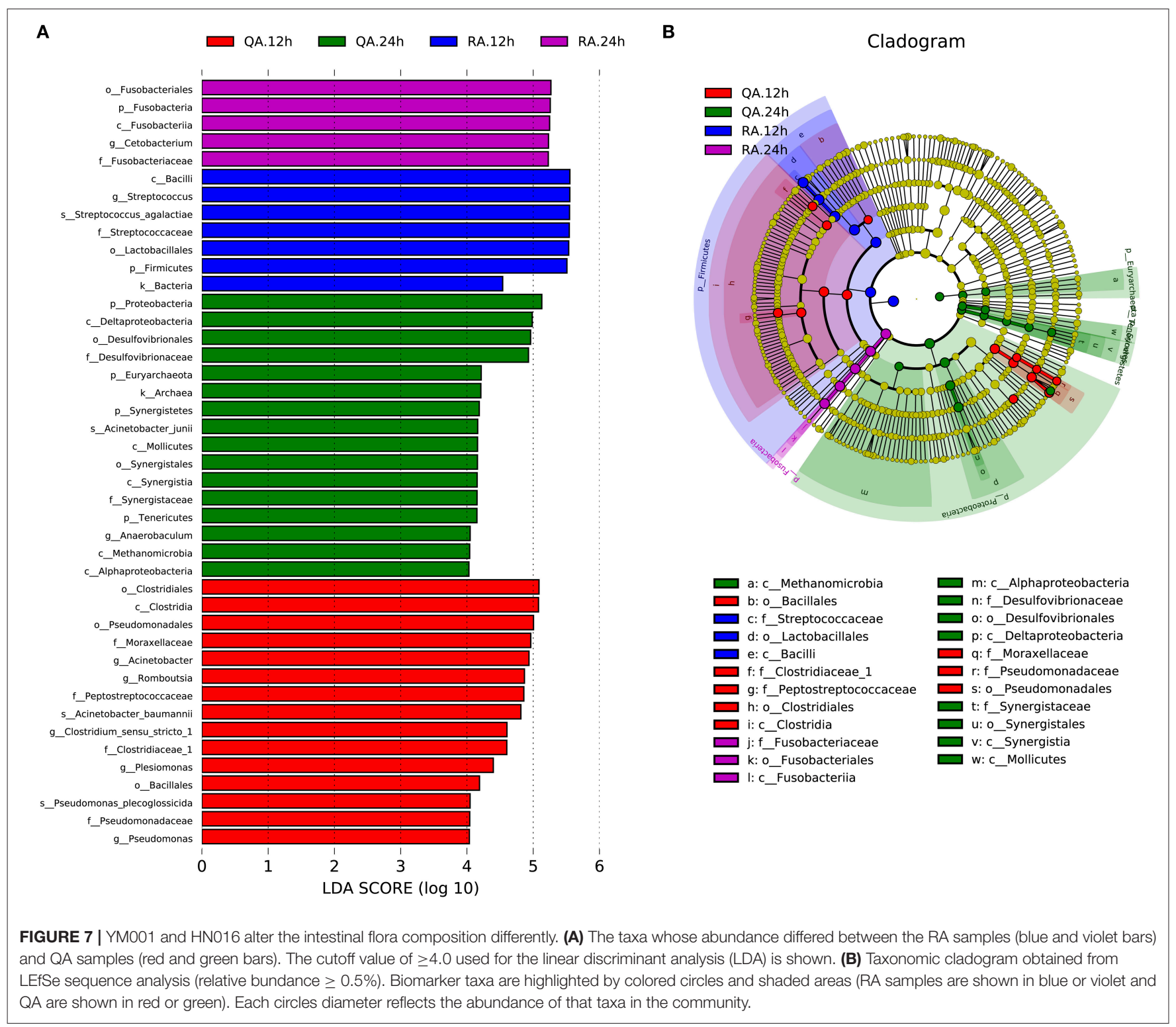

found in almost every segment of the digestive tract. At the same time, unique colonies are formed in various segments of the digestive tract. To our best knowledge, there are no reports about the bacterial structures in different segments of the digestive tract in tilapia. We found that there are significant differences in the microbial composition at different segments of the digestive tract of tilapia (Figures 2A,B). The differences in microbiota at different parts of segments of the digestive tract are closely related to their physiological characteristics.

The diversity and abundance of intestinal microbiota in animals may change due to factors such as age, dietary changes, medication, and infection (Xia et al., 2014). One instance is that canine distemper virus infection distorted the intestinal microbiota composition by reducing the prevalence of the dominant genera, Escherichia and Clostridium, and increasing microbial diversity. In this study, we performed high-throughput
16S RNA gene sequencing of the intestinal microbiota of tilapia after oral administration of $S$. agalactiae YM001. Through $\beta$ diversity analysis, it was found that early oral YM001 immunization could decrease intestinal microbiota diversity in tilapia (Figure 3A). Changes in intestinal microbiota may have an impact on the health of the host. Previous studies have shown that changes in intestinal microbiota may have an impact on the health of the host. For human, the decrease in bacterial richness and diversity is consistent with other disease states that have alterations in the intestinal microbiota such as inflammatory bowel disease and diabetes (Jandhyala et al., 2017; Nishino et al., 2018). Despite this, administration of YM001 only temporarily reduced the diversity of intestinal microbiota in tilapia (Figures 3A,B) and changes in intestinal microbial diversity in tilapia appear to be closely related to YM001 concentration in the intestine. By analyzing the 
relationship between the retention of oral streptococcus and the diversity of intestinal microbiota, we found that when the concentration of YM001 was highest, the diversity of intestinal microbiota in tilapia was the lowest, and with the concentration of YM001 decreasing, the diversity was gradually recovered (Figures 3A,B). Oral administration of virulent strain HN016 also decreased the intestinal microbiota diversity of tilapia, but as the concentration of HN016 decreasing, the intestinal microbiota diversity also recovered or even exceeded the normal level (Figures 6A,B). More and more scholars started to explore the effects of oral vaccines on intestinal microbiota in recent years. It has been reported that oral administration of live-attenuated typhoid vaccine Ty21a could considerably affect inter- and intraindividual variability, yet not discernibly perturb the bacterial assemblage related to vaccine administration (Eloe-Fadrosh et al., 2013). Oral administration of different $S$. Typhimurium vaccine strains can differentially influence the presence, but not the relative abundance of microbiota in the ceca (Park et al., 2017). Similar studies have also been reported in aquatic animals. When bath-immunizing grass carp using recombinant Aeromonas hydrophila vaccine (Aera), there was no significant difference in the abundance of genera Acinetobacter, Cetobacterium, and Gemmobacter compared to the Control (Liu et al., 2015). All the above examples indicate that oral vaccines can affect the composition of the intestinal microbiota to varying degrees.

Intestinal microbiota imbalance could cause host health problems, which may be related to reduced levels of beneficial bacteria and increased levels of pathogenic bacteria. By analyzing the microbiota changes in the different gut segments, it was found that at the genus level, the contents of Streptococcus, Cetobacterium, Akkermansia, Romboutsia, Bacteroides, Brevinema and Lachnospiraceae_NK4A136 are changed the most after administration of YM001 (Figure 4A). Ecept the Streptococcus which are directly related to YM001, the function of the other above bacteria are as follow. Cetobacterium is bile resistant and could produce acetic acid as the major end product of metabolism of peptides and carbohydrates (Finegold et al., 2003). A. muciniphila is a mucin-degrading bacterium and inversely associated with obesity, diabetes, inflammation, and metabolic disorders (Everard et al., 2013; Caesar et al., 2015). Romboutsia can utilize different relatively simple carbohydrates and synthesize limited amino acids and vitamins (Gerritsen et al., 2017). Bacteroides play a fundamental role in processing complex molecules to simpler ones in the host intestine (Wexler, 2007). Bacteroides species also benefit their host by excluding potential pathogens from colonizing the gut (Wexler, 2007). Some species (B. fragilis, for example) are opportunistic human pathogens (Wexler, 2007). Brevinema belongs to spirochaetes, which have been extensively studied in termite guts and have been found to be involved in the breakdown of lignocellulose and nitrogen fixation. Lachnospiraceae are a family of bacteria in

\section{REFERENCES}

Amal, M. N., Zamri-Saad, M., Iftikhar, A. R., Siti-Zahrah, A., Aziel, S., and Fahmi, S. (2012). An outbreak of Streptococcus agalactiae infection in cage-cultured golden pompano, Trachinotus blochii (Lacepede), the order of Clostridiales. Members of Lachnospiraceae family could produce butyric acid and are important for host epithelial cell growth (Meehan and Beiko, 2014). Among these bacteria, the change of Cetobacterium is particularly noteworthy. As the predominant bacteria in the intestine of tilapia, Cetobacterium accounted for $24.87 \%$ of the total intestinal bacteria, and its content increased to $41.1 \%$ at $24 \mathrm{~h}$ after oral administration of YM001, and gradually returned to 32.2 and $29 \%$ at $7 \mathrm{~d}$ and 15 $\mathrm{d}$ after oral administration of YM001. Streptococcus accounted for $0.5 \%$ of the total intestinal bacteria in healthy tilapia. At $12 \mathrm{~h}$ after oral administration of YM001, its content increased to up to $70.8 \%$ and then gradually returned to $0.8 \%$ at $24 \mathrm{~h}$ after oral administration of YM001. The results showed that oral YM001 changed the composition of intestinal microbiota in a short time; but with the decrease of YM001 content, the content of the predominant bacteria was gradually recovered.

The effects of attenuated strain YM001 and virulent strain HN016 on tilapia intestinal microbiota were very different. The differences in gut bacteria between these two groups were greater than the differenced within each group (Figure 5). Although oral administration of HN016 and YM001 both decreased the intestinal flora diversity, the effect of YM001 was more significant (Figure 6). The difference in intestinal flora caused by virulent strains and attenuated strains may be related to the secretion or metabolites of different strains. The specific underlying mechanisms need to be further explored.

In conclusion, after oral administration, YM001 could colonize in the digestive tract of tilapia for a certain period of time, and alter the structure and diversity of the intestinal microbiota. However, these changes do not have fatal consequences. Tilapia intestinal microbiota has a repulsive effect against YM001. With the content of YM001 in the digestive decreasing, the structure and diversity of intestinal microbiota gradually recovered.

\section{AUTHOR CONTRIBUTIONS}

ML, LL, and TH contributed equally to this work. LL, TH, YL, $\mathrm{AL}$, and FC performed experiments and analyzed data. ML and CM provided software and bioinformatics expertise. MC and ML designed the experiments, analyzed data, and wrote the manuscript.

\section{FUNDING}

This work was supported by Guangxi innovation-driven development special funds (Grant no. AA17204081-3), National Natural Science Foundation of China (31460695), Guangxi Natural Science Foundation (2016GXNSFDA380020), the funds of Guangxi Key Laboratory for Aquatic Genetic Breeding and Healthy Aquaculture (2016-2018).

in Malaysia. J. Fish Dis. 35, 849-852. doi: 10.1111/j.1365-2761.2012. 01443.x

Baquero, F., Martinez, J. L., and Canton, R. (2008). Antibiotics and antibiotic resistance in water environments. Curr. Opin. Biotechnol. 19, 260-265. doi: 10.1016/j.copbio.2008.05.006 
Barroso-Batista, J., Demengeot, J., and Gordo, I. (2015). Adaptive immunity increases the pace and predictability of evolutionary change in commensal gut bacteria. Nat. Commun. 6:8945. doi: 10.1038/ncomms9945

Caesar, R., Tremaroli, V., Kovatcheva-Datchary, P., Cani, P. D., and Backhed, F. (2015). Crosstalk between gut microbiota and dietary lipids aggravates WAT inflammation through TLR signaling. Cell Metab. 22, 658-668. doi: 10.1016/j.cmet.2015.07.026

Chen, M., Wang, R., Li, L. P., Liang, W. W., Li, J., Huang, Y., et al. (2012). Screening vaccine candidate strains against Streptococcus agalactiae of tilapia based on PFGE genotype. Vaccine 30, 6088-6092. doi: 10.1016/j.vaccine.2012. 07.044

Chen, W. H., Garza, J., Choquette, M., Hawkins, J., Hoeper, A., Bernstein, D. I., et al. (2015). Safety and immunogenicity of escalating dosages of a single oral administration of peru-15 pCTB, a candidate live, attenuated vaccine against enterotoxigenic Escherichia coli and Vibrio cholerae. Clin. Vaccine Immunol. 22, 129-135. doi: 10.1128/CVI.00560-14

Depaola, A., Peeler, J. T., and Rodrick, G. E. (1995). Effect of oxytetracyclinemedicated feed on antibiotic resistance of gram-negative bacteria in catfish ponds. Appl. Environ. Microbiol. 61, 2335-2340.

Dethlefsen, L., Eckburg, P. B., Bik, E. M., and Relman, D. A. (2006). Assembly of the human intestinal microbiota. Trends Ecol. Evol. 21, 517-523. doi: $10.1016 /$ j.tree.2006.06.013

Edmond, K. M., Kortsalioudaki, C., Scott, S., Schrag, S. J., Zaidi, A. K., Cousens, S., et al. (2012). Group B streptococcal disease in infants aged younger than 3 months: systematic review and meta-analysis. Lancet 379, 547-556. doi: 10.1016/S0140-6736(11)61651-6

Edwards, M. S., and Baker, C. J. (2005). Group B streptococcal infections in elderly adults. Clin. Infect. Dis. 41, 839-847. doi: 10.1086/432804

Eloe-Fadrosh, E. A., Mcarthur, M. A., Seekatz, A. M., Drabek, E. F., Rasko, D. A., Sztein, M. B., et al. (2013). Impact of oral typhoid vaccination on the human gut microbiota and correlations with s. Typhi-specific immunological responses. PLoS ONE 8:e62026. doi: 10.1371/journal.pone.00 62026

Everard, A., Belzer, C., Geurts, L., Ouwerkerk, J. P., Druart, C., Bindels, L. B., et al. (2013). Cross-talk between Akkermansia muciniphila and intestinal epithelium controls diet-induced obesity. Proc. Natl. Acad. Sci. U.S.A. 110, 9066-9071. doi: $10.1073 /$ pnas. 1219451110

Finegold, S. M., Vaisanen, M. L., Molitoris, D. R., Tomzynski, T. J., Song, Y., Liu, C., et al. (2003). Cetobacterium somerae sp. nov. from human feces and emended description of the genus Cetobacterium. Syst. Appl. Microbiol. 26, 177-181. doi: 10.1078/072320203322346010

Gaikwad, S. S., Shouche, Y. S., and Gade, W. N. (2017). Deep sequencing reveals highly variable gut microbial composition of invasive fish mossambicus tilapia (Oreochromis mossambicus) collected from two different habitats. Indian J. Microbiol. 57, 235-240. doi: 10.1007/s12088-017-0641-9

Gerritsen, J., Hornung, B., Renckens, B., Van Hijum, S., Martins Dos Santos, V. A. P., and Smidt, H. (2017). Genomic and functional analysis of Romboutsia ilealis CRIB(T) reveals adaptation to the small intestine. PeerJ. 5:e3698. doi: $10.7717 /$ peerj.3698

Gómez, G. D., and Balcazar, J. L. (2008). A review on the interactions between gut microbiota and innate immunity of fish. FEMS Immunol. Med. Microbiol. 52, 145-154. doi: 10.1111/j.1574-695X.2007.00343.x

Harris, V. C., Armah, G., Fuentes, S., Korpela, K. E., Parashar, U., Victor, J. C., et al. (2017). Significant correlation between the infant gut microbiome and rotavirus vaccine response in rural ghana. J. Infect. Dis. 215, 34-41. doi: 10.1093/infdis/jiw518

Hopkins, M. J., Sharp, R., and Macfarlane, G. T. (2002). Variation in human intestinal microbiota with age. Dig. Liver Dis. 34(Suppl. 2):S12-18. doi: 10.1016/S1590-8658(02)80157-8

Huang, L. Y., Wang, K. Y., Xiao, D., Chen, D. F., Geng, Y., Wang, J., et al. (2014). Safety and immunogenicity of an oral DNA vaccine encoding Sip of Streptococcus agalactiae from Nile tilapia Oreochromis niloticus delivered by live attenuated Salmonella typhimurium. Fish Shellfish Immunol. 38, 34-41. doi: 10.1016/j.fsi.2014.02.017

Jafar, Q. A., Sameer, A. Z., Salwa, A. M., Samee, A. A., Ahmed, A. M., and AlSharifi, F. (2008). Molecular investigation of Streptococcus agalactiae isolates from environmental samples and fish specimens during a massive fish kill in Kuwait Bay. Pak. J. Biol. Sci. 11, 2500-2504. doi: 10.3923/pjbs.2008.2500.2504
Jandhyala, S. M., Madhulika, A., Deepika, G., Rao, G. V., Reddy, D. N., Subramanyam, C., et al. (2017). Altered intestinal microbiota in patients with chronic pancreatitis: implications in diabetes and metabolic abnormalities. Sci. Rep. 7:43640. doi: 10.1038/srep43640

Kelly, P. (2010). Nutrition, intestinal defence and the microbiome. Proc. Nutr. Soc. 69, 261-268. doi: 10.1017/S0029665110000108

Lamagni, T. L., Keshishian, C., Efstratiou, A., Guy, R., Henderson, K. L., Broughton, K., et al. (2013). Emerging trends in the epidemiology of invasive group B streptococcal disease in England and Wales, 1991-2010. Clin. Infect. Dis. 57, 682-688. doi: 10.1093/cid/cit337

Ley, R. E., Lozupone, C. A., Hamady, M., Knight, R., and Gordon, J. I. (2008). Worlds within worlds: evolution of the vertebrate gut microbiota. Nat. Rev. Microbiol. 6, 776-788. doi: 10.1038/nrmicro1978

Li, L. P., Wang, R., Liang, W. W., Huang, T., Huang, Y., Luo, F. G., et al. (2015). Development of live attenuated Streptococcus agalactiae vaccine for tilapia via continuous passage in vitro. Fish Shellfish Immunol. 45, 955-963. doi: 10.1016/j.fsi.2015.06.014

Liu, L., Gong, Y. X., Zhu, B., Liu, G. L., Wang, G. X., and Ling, F. (2015). Effect of a new recombinant Aeromonas hydrophila vaccine on the grass carp intestinal microbiota and correlations with immunological responses. Fish Shellfish Immunol. 45, 175-183. doi: 10.1016/j.fsi.2015. 03.043

Makesh, M., Sudheesh, P. S., and Cain, K. D. (2015). Systemic and mucosal immune response of rainbow trout to immunization with an attenuated Flavobacterium psychrophilum vaccine strain by different routes. Fish Shellfish Immunol. 44, 156-163. doi: 10.1016/j.fsi.2015. 02.003

Meehan, C. J., and Beiko, R. G. (2014). A phylogenomic view of ecological specialization in the Lachnospiraceae, a family of digestive tract-associated bacteria. Genome Biol. Evol. 6, 703-713. doi: 10.1093/gbe/evu050

Mian, G. F., Godoy, D. T., Leal, C. A., Yuhara, T. Y., Costa, G. M., and Figueiredo, H. C. (2009). Aspects of the natural history and virulence of S. agalactiae infection in Nile tilapia. Vet. Microbiol. 136, 180-183. doi: 10.1016/j.vetmic.2008.10.016

Nishino, K., Nishida, A., Inoue, R., Kawada, Y., Ohno, M., Sakai, S., et al. (2018). Analysis of endoscopic brush samples identified mucosa-associated dysbiosis in inflammatory bowel disease. J. Gastroenterol. 53, 95-106. doi: 10.1007/s00535-017-1384-4

O'mahony, S. M., Marchesi, J. R., Scully, P., Codling, C., Ceolho, A. M., Quigley, E. M., et al. (2009). Early life stress alters behavior, immunity, and microbiota in rats: implications for irritable bowel syndrome and psychiatric illnesses. Biol. Psychiatry 65, 263-267. doi: 10.1016/j.biopsych.2008. 06.026

Park, S. H., Kim, S. A., Rubinelli, P. M., Roto, S. M., and Ricke, S. C. (2017). Microbial compositional changes in broiler chicken cecal contents from birds challenged with different Salmonella vaccine candidate strains. Vaccine 35, 3204-3208. doi: 10.1016/j.vaccine.2017.04.073

Pridgeon, J. W., and Klesius, P. H. (2013). Development of live attenuated Streptococcus agalactiae as potential vaccines by selecting for resistance to sparfloxacin. Vaccine 31, 2705-2712. doi: 10.1016/j.vaccine.2013. 03.066

Rawls, J. F., Samuel, B. S., and Gordon, J. I. (2004). Gnotobiotic zebrafish reveal evolutionarily conserved responses to the gut microbiota. Proc. Natl. Acad. Sci. U.S.A. 101, 4596-4601. doi: 10.1073/pnas.0400706101

Roeselers, G., Mittge, E. K., Stephens, W. Z., Parichy, D. M., Cavanaugh, C. M., Guillemin, K., et al. (2011). Evidence for a core gut microbiota in the zebrafish. ISME J. 5, 1595-1608. doi: 10.1038/ismej.2011.38

Schmidt, B., Mulder, I. E., Musk, C. C., Aminov, R. I., Lewis, M., Stokes, C. R., et al. (2011). Establishment of normal gut microbiota is compromised under excessive hygiene conditions. PLoS ONE 6:e28284. doi: 10.1371/journal.pone.0028284

Sommer, F., and Backhed, F. (2013). The gut microbiota-masters of host development and physiology. Nat. Rev. Microbiol. 11, 227-238. doi: 10.1038/nrmicro2974

Tanaka, S., Kobayashi, T., Songjinda, P., Tateyama, A., Tsubouchi, M., Kiyohara, C., et al. (2009). Influence of antibiotic exposure in the early postnatal period on the development of intestinal microbiota. FEMS Immunol. Med. Microbiol. 56, 80-87. doi: 10.1111/j.1574-695X.2009.00553.x 
Tazi, A., Morand, P. C., Reglier-Poupet, H., Dmytruk, N., Billoet, A., Antona, D., et al. (2011). Invasive group B streptococcal infections in adults, France (2007-2010). Clin. Microbiol. Infect. 17, 1587-1589. doi: 10.1111/j.1469-0691.2011.03628.x

Turnbaugh, P. J., Hamady, M., Yatsunenko, T., Cantarel, B. L., Duncan, A., Ley, R. E., et al. (2009). A core gut microbiome in obese and lean twins. Nature 457, 480-484. doi: 10.1038/nature07540

Wang, R., Li, L., Huang, Y., Luo, F., Liang, W., Gan, X., et al. (2015). Comparative genome analysis identifies two large deletions in the genome of highly-passaged attenuated Streptococcus agalactiae strain YM001 compared to the parental pathogenic strain HN016. BMC Genom. 16:897. doi: 10.1186/s12864-015-2026-y

Wexler, H. M. (2007). Bacteroides: the good, the bad, and the nitty-gritty. Clin. Microbiol. Rev. 20, 593-621. doi: 10.1128/CMR. 00008-07
Xia, J. H., Lin, G., Fu, G. H., Wan, Z. Y., Lee, M., Wang, L., et al. (2014). The intestinal microbiome of fish under starvation. BMC Genom. 15:266. doi: $10.1186 / 1471-2164-15-266$

Conflict of Interest Statement: The authors declare that the research was conducted in the absence of any commercial or financial relationships that could be construed as a potential conflict of interest.

Copyright (C) $2019 \mathrm{Li}, \mathrm{Li}$, Huang, Liu, Lei, Ma, Chen and Chen. This is an open-access article distributed under the terms of the Creative Commons Attribution License (CC $B Y)$. The use, distribution or reproduction in other forums is permitted, provided the original author(s) and the copyright owner(s) are credited and that the original publication in this journal is cited, in accordance with accepted academic practice. No use, distribution or reproduction is permitted which does not comply with these terms. 